\title{
OPEN The metabolic cost of turning right side up in the Mediterranean spur-thighed tortoise (Testudo graeca)
}

\author{
Heather E. Ewart $\mathbb{1}^{1}$, Peter G. Tickle $\mathbb{1}^{2}$, William I. Sellers $\mathbb{1}^{3}$, Markus Lambertz $\mathbb{1}^{4,5}$, \\ Dane A. Crossley II ${ }^{6}$ \& Jonathan R. Codd $1{ }^{1 \bowtie}$
}

Armoured, rigid bodied animals, such as Testudines, must self-right should they find themselves in an inverted position. The ability to self-right is an essential biomechanical and physiological process that influences survival and ultimately fitness. Traits that enhance righting ability may consequently offer an evolutionary advantage. However, the energetic requirements of self-righting are unknown. Using respirometry and kinematic video analysis, we examined the metabolic cost of self-righting in the terrestrial Mediterranean spur-thighed tortoise and compared this to the metabolic cost of locomotion at a moderate, easily sustainable speed. We found that self-righting is, relatively, metabolically expensive and costs around two times the mass-specific power required to walk. Rapid movements of the limbs and head facilitate successful righting however, combined with the constraints of breathing whilst upside down, contribute a significant metabolic cost. Consequently, in the wild, these animals should favour environments or behaviours where the risk of becoming inverted is reduced.

Testudines are unique among extant amniotes in possessing a bony shell. The evolution of a fully ossified shell was only possible due to a flank-muscle driven ventilatory apparatus that evolved around 50 million-years prior ${ }^{1}$. The shell is formed from the vertebral column, expanded ribs and gastralia that, with the inferred loss of the intercostal muscles ${ }^{1}$, prevents breathing using expansion and contraction of the rib cage. Tortoise inspiration and expiration is predominantly controlled by a 'muscular sling' involving two muscles; the M. obliquus abdominis and $M$. transversus abdominis ${ }^{1}$. These novel adaptations in abdominal muscle function had to have occurred in the ancestors of turtles long before they evolved their shells ${ }^{1,2}$. Once ventilation no longer required flexibility of the trunk, a constraint on the dorsal ribs was removed, allowing them to further broaden, fuse and eventually form a rigid carapace ${ }^{1}$. Although the method of breathing is strikingly different in turtles they are reported to have a cost of breathing, the energy expended to power the respiratory system, similar to other vertebrates ${ }^{3}$. The cost or work of breathing will impact all aspects of an animal's daily energy budget including the energetic cost of moving. Breathing in turtles is not correlated to gait cycle during terrestrial locomotion, suggesting a de-coupling of respiration and locomotion ${ }^{4}$.

Shells convey a range of advantages for tortoises including improved protection from predation and environmental stressors ${ }^{5-7}$. However, the trade-offs for having an inflexible vertebral column and rib cage include its influences on tortoise movement and locomotion ${ }^{1}$. Tortoises are famous for their slow locomotion, which is directly related to their rigid and inflexible bodies ${ }^{8}$. Tortoises locomote with diagonally opposite feet moving together so that two feet are in contact with the ground at almost all times ${ }^{9}$. These adjustment in gait are thought to limit body swaying and enable them to avoid fast and energetically expensive movements of the limbs ${ }^{9}$. The metabolic cost of locomotion (CoT) has been directly measured using respiromety in only two Testudines species, Emydura macquarii and Terrapene ornata ${ }^{10}$. In both these species the CoT was around half of that expected ${ }^{11}$. Tortoise locomotion is notably more energetically efficient than that of other vertebrates of similar mass due in part to their limb muscle physiology ${ }^{12,13}$. The use of only one gait (walking) over their entire limited speed range during locomotion allows the maximum optimisation for energy efficiency of slow muscle fibres ${ }^{13}$.

\footnotetext{
${ }^{1}$ School of Biological Sciences, University of Manchester, Manchester, UK. ${ }^{2}$ School of Biomedical Sciences, University of Leeds, Leeds, UK. ${ }^{3}$ School of Natural Sciences, University of Manchester, Manchester, UK. ${ }^{4}$ Institut Für Zoologie, Rheinische Friedrich-Wilhelms-Universität Bonn, Bonn, Germany. ${ }^{5}$ Sektion Herpetologie, Zoologisches Forschungs Museum Alexander Koenig, Bonn, Germany. ${ }^{6}$ Department of Biological Sciences, University of North Texas, Denton, USA. ${ }^{\square}$ email: jonathan.codd@manchester.ac.uk
} 
Aside from the impacts on how they breathe, being limited by their mobility, rigid-bodied and armoured terrestrial Testudines species are also susceptible to overturning through every-day occurrences such as competition, mating behaviour, predation attempts, or locomotion over uneven landscapes ${ }^{14,15}$. Failure to self-right once turned upside-down can lead to life-threatening consequences including predation, starvation, desiccation, loss of mating or foraging opportunities, and thermal stress ${ }^{14-18}$. Within the Testudines there are two strategies for self-righting; (i) using neck extension to rapidly push the body around its longitudinal axis to an upright position $^{19}$, or (ii) when the neck does not extend sufficiently to raise the body above the ground, aggressively shaking the limbs and head to create the momentum to spin the body to an angle at which they can flip to an upright position (Supplementary Movie S1). Research into self-righting has focussed primarily on the influence of body size, body shape and how morphological variations in limb lengths or measures of flexibility habitat structure and ruggedness affect righting ability ${ }^{15,19-22}$. These studies have demonstrated that righting ability is primarily related to carapace structure, shape and size as well as the extension length of the limbs, including the neck $^{19}$. Recent studies have developed theoretical models of the interaction between shell shape and righting ${ }^{19,23,24}$ and the influence of biotic or abiotic factors ${ }^{25}$. Self-righting performance is commonly quantified as the total time taken to right ${ }^{26}$ and is considered an important fitness-related trait ${ }^{12,27}$. Fitness proxies often centre around carapace morphology and modelling of the energetics of righting ${ }^{27}$ and have been used to link self-righting performance to improved survival ${ }^{28}$. However, the actual metabolic cost of self-righting, which is an essential element for assessing fitness, has never been experimentally quantified in any Testudines.

Accordingly, using respirometry and video analysis, we measured the metabolic cost of self-righting (CoR) in female sub-adult Mediterranean spur-thighed tortoise (Testudo graeca) that uses movements of the limbs and head to self-right. We placed the CoR into context by comparison to the metabolic cost of transport (CoT) at an average sustainable speed.

\section{Results}

The tortoises we tested were unable to self-right on a hard surface; however, through a set of repetitive movements, the tortoises could effectively turn right side up on a granular substrate (Supplementary Movie S1). Once inverted the tortoises will begin a characteristic set of movements and always self-right via a transversal roll over the longitudinal axis ${ }^{19}$. Rapid waggling (bobbing-lateral movements) of the head, and swinging (lateral movements) of the front and hind limbs begins immediately once inverted. These rapid limb movements, the limbs and head come into contact with the ground which have the effect of turning and rotating the body around in a circular motion, while gouging out a channel in the substrate. As the tortoise creates a channel, often completing a half or full circle, the body is angled to a degree that will allow the limbs closest to the substrate and the head to come into contact with the ground through a series of swinging movements. These movements have the effect of deepening the channel and accompanying mound behind the body against which the tortoise can push the carapace.

It is finally through the use of these swinging movements that the tortoise is able to create a channel deep enough to reach the tipping point of the angle of the carapace against the substrate, that the animal will flip (Supplementary Movie S1).

Respirometry demonstrated that the $\mathrm{CoR}$ is relatively energetically expensive $\left(\dot{V} \mathrm{O}_{2} 0.66 \pm 0.09 \mathrm{~mL} \mathrm{~min}^{-1}\right.$, $\left.\mathrm{P}_{\text {met }} 33.59 \pm 3.61 \mathrm{~W} \mathrm{~kg}^{-1}\right)$, at over 2 times $\operatorname{CoT}\left(\dot{V} \mathrm{O}_{2} 0.34 \pm 0.04 \mathrm{~mL} \mathrm{~min}^{-1}, \mathrm{P}_{\text {met }} 19.71 \pm 1.26 \mathrm{~W} \mathrm{~kg}^{-1}\right)$ and four to five-fold greater than resting metabolic rate (RMR) $\left(\dot{V} \mathrm{O}_{2} 0.15 \pm 0.02 \mathrm{ml} \mathrm{min}^{-1}, \mathrm{P}_{\text {met }} 8.27 \pm 0.91 \mathrm{~W} \mathrm{~kg}^{-1}\right)$ (Fig. 1a,b, Supplementary Tables S1, S2). Interestingly, kinematic analyses showed maximum limb movement frequency during righting $(13.99 \pm 4.10 \mathrm{~Hz}$, Supplementary Movie S1) was five-fold higher than that during walking at a speed of $6 \mathrm{~cm} \mathrm{~s}^{-1}(2.72 \pm 0.31 \mathrm{~Hz}$, Supplementary Movie S2). In addition, there was also a link between total righting duration time $(12.6 \pm 2.02 \mathrm{~min})$ and the power requirement, with the $\mathrm{P}_{\text {met }}$ increasing linearly with the time taken to right (Fig. 1c). All data mean \pm SE.

\section{Discussion}

Self-righting is an intriguing behaviour in Testudines and one that is important for understanding such fundamental aspects of their biology as the evolution of their shell shape $e^{12,19}$, survivability of hatchlings ${ }^{28}$. The shells of Testudines are an evolutionary novelty. The rigid shell is a relatively fixed volume, which means that anything that alters the space available inside the shell could restrict lung volume and ultimately influence breathing. Despite having the pelvic and pectoral girdles inside the shell, through their specialised abdominal ventilatory mechanism, terrestrial Testudines locomotion performance does not appear to conflict with breathing performance ${ }^{29}$. However, our data indicate that self-righting incurs a significant energetic expense, relative to walking, that suggests there may be an as yet unconsidered novel constraints associated with having evolved a shell. During inspiration the action of the oblique muscle is aided indirectly by a gravity-induced ventral pull on the liver, that facilitates inflation of the lungs ${ }^{30}$. However, being upside down reverses the direction of gravity acting on the liver. Coupled with the increased mass of the viscera, predominantly the stomach that along with the liver is directly connected via inter-organ connections to the ventral side of the lungs ${ }^{31}$, being inverted likely constricts the ability of the lung to inflate thereby reducing lung volume or at the very least decreases pulmonary compliance. Submersion in aquatic species has an analogous effect in reducing lung volume and ultimately increases the work of breathing ${ }^{32}$. Any increases in the work of breathing would contribute to the metabolic cost of self-righting. Although being inverted would act to reduce lung volume, the post pulmonary septum (PPS, Fig. 2) likely prevents total collapse of the lung. Interestingly the PPS is reduced in species such as the common snapping turtle (Chelydra serpentina) ${ }^{31}$ that use rapid neck driven self-righting ${ }^{5}$, suggesting future research into any functional linkage between degree of lung coverage by the PPS and the adoption of a self-righting strategy would be beneficial. 


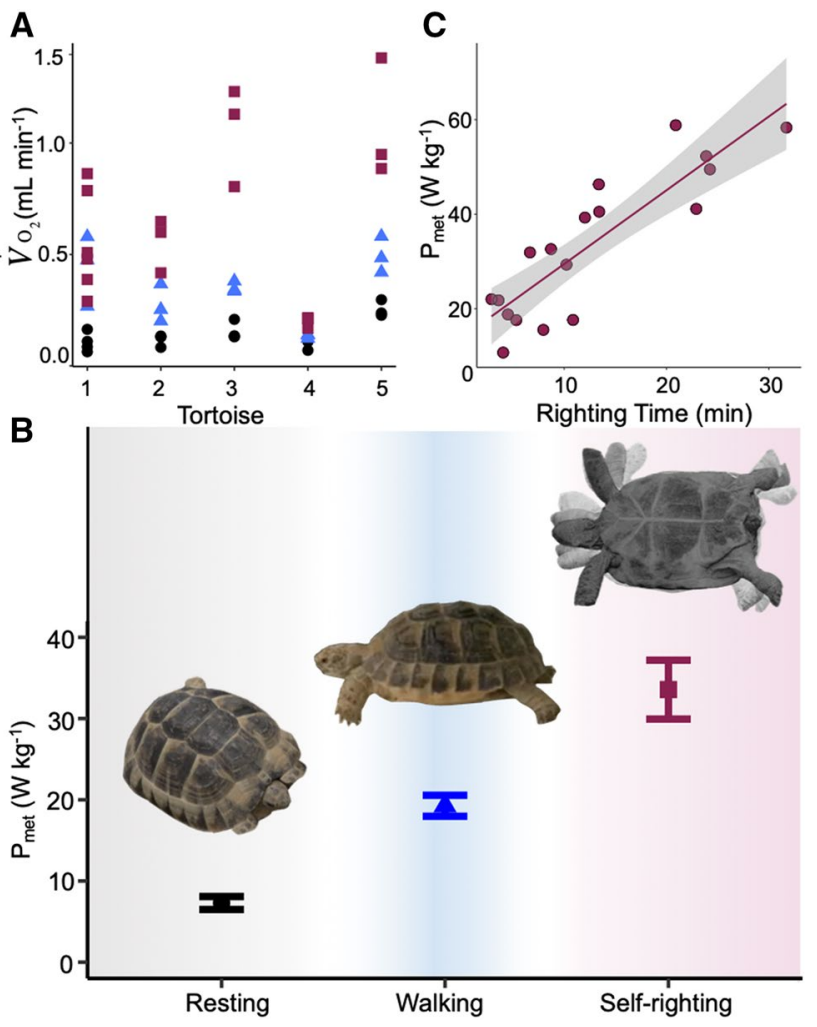

Figure 1. The cost of self-righting and walking (A) Rate of oxygen consumption $\left(\dot{V} \mathrm{O}_{2}, \mathrm{~mL} \mathrm{~min}^{-1}\right)$ and (B) Mass specific metabolic power $\left(\mathrm{P}_{\text {met, }} \mathrm{W} \mathrm{kg}^{-1}\right.$ ) during resting (black circles), walking (blue triangles) and selfrighting (maroon squares). Self-righting is the most metabolically expensive behaviour using over two times the oxygen and mass specific metabolic power per $\mathrm{kg}$ compared to walking at $6 \mathrm{~cm} \mathrm{~s}^{-1}$ and four to five-fold that at resting. Representative images are snapshots representing the stages correlating to resting, walking and the self-righting process for the Mediterranean spur-thighed tortoise (Supplementary Movie S1). (C) Mass-specific metabolic power $\left(\mathrm{P}_{\mathrm{met}}, \mathrm{W} \mathrm{kg}{ }^{-1}\right)$ of self-righting tortoises plotted against time $(\mathrm{min})$, demonstrating the linear increase with total time taken to self-right. Shaded region represents the $95 \%$ confidence interval. All data mean \pm SE. Tortoise images taken by HE.

The energetic cost of behaviours relevant for free-ranging animals is often overlooked, including how these might vary during ontogeny, however, these studies provide a crucial link between physiology and ecology $\mathrm{y}^{33}$. The cost of movement can be a substantial and ultimately any energy used for movement isn't available for other functions, creating a trade-off against the energy available for growth, feeding, thermoregulation and/or reproduction ${ }^{33,34}$. Therefore, each of these components must be serviced while attempting to ensure that overall energy output $\leq$ energy input. Energy input is not infinite and metabolic scope (the difference between resting and maximal metabolic rates) is limited under real conditions ${ }^{34}$. Given the option animals will tend to make decisions that minimise their energy output for a given $\operatorname{task}^{35}$ and will make appropriate choices on how and where to move within an energetic landscape ${ }^{36}$ suggesting that for tortoises they should seek to minimise the energy being expended by avoiding inverting. Generally, there are very few studies examining the energetics of any behaviours in Testudines. The metabolic cost of transport, for example, has only been examined in two species (prior to the single speed reported in the current study) in a terrestrial (Cryptodira: Terrapene ornata ${ }^{10}$ ) and aquatic (Pleurodira: Emdura macquari ${ }^{37}$ ) species. Both these studies demonstrated that metabolic cost of locomotion was surprisingly lower than expected for their body mass and when compared to other amniotes ${ }^{10,37}$. Our data support the previous findings that testudines are able to move very efficiently.

Locomotion is a significant component of the daily activity and energy budgets of most animals, including tortoises ${ }^{38}$. The CoT has been studied across a wide range of animals and is primarily determined by the energetic cost of generating muscle force to support and propel body weight ${ }^{39}$. CoT is optimised by increasing the metabolic efficiency of muscle contractions and by minimising the mechanical work done during locomotion $^{40,41}$. The unique anatomy of the Testudines has eliminated their need for the muscular sling found in other quadrupeds ${ }^{42}$, which may serve as an energy saving mechanism during their movement ${ }^{10}$. Slow moving tortoise terrestrial locomotion is more efficient than that of other vertebrates of a similar mass, due in part to their muscle physiology $y^{11,13,43}$. The predominance of type I fibres in tortoise locomotor muscles, that are known to stretch and contract at a slower rate than other vertebrates ${ }^{13}$, is adaptive for lower intensity movements such as walking. Slow contracting tortoise muscles are in fact the most efficient ever studied, with $35 \%$ of available chemical energy converted to mechanical work during contraction ${ }^{13}$; this compares to thermodynamic efficiency 

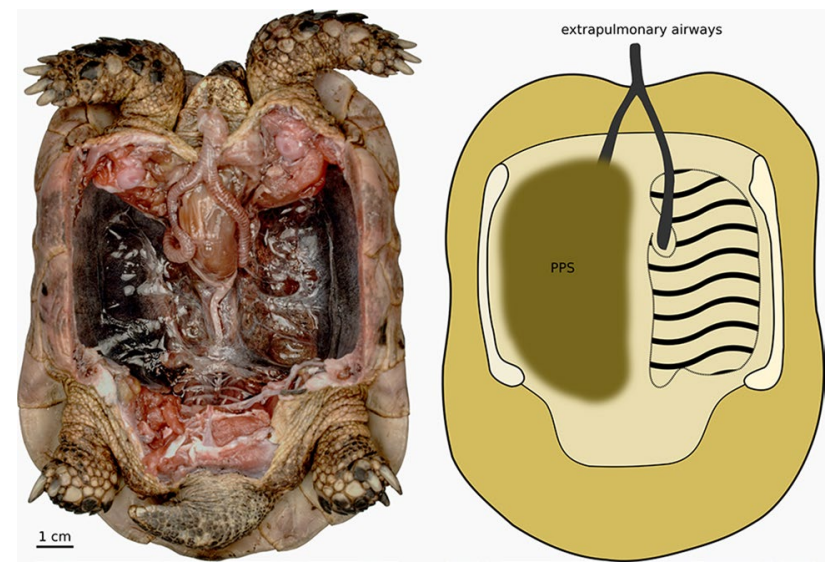

Figure 2. The Post Pulmonary Septum in Testudo Overview of the coelomic subdivision and the resulting compartmentalization of the respiratory system in tortoises. The left image shows in a ventral view the viscera, except for the lungs, have been removed. The schematic drawing on the right indicates the structures in question. Unlike in most other clades of chelonians, the ventral side of the lungs is completely covered by the postpulmonary septum (PPS). The anatomically left side shows the medial parts of the PPS removed, giving a clear view on the actual lungs beneath (i.e. dorsal to) it. Dorsally, the lungs are broadly fused to the inner side of the carapace (indicated by the wavy lines), resulting in a firm fixation within the pleural cavity. Note the PPS extending laterad to the carapace. The anatomical configuration serves as a mechanical support system in inverted tortoises whose viscera are pushing on the lungs. As a consequence, the PPS prevents gravitational pulmonary collapse in inverted specimens and hence counteracts the resulting decrease in compliance together with the associated increase in work of breathing. Photograph taken by ML.

of only c. $20 \%$ in mouse and dogfish muscle ${ }^{44}$. The energetic efficiency of muscle is inversely related to shortening speed $^{45}$, with force generation notably more efficient in slow twitch type I muscle fibres compared to fast twitch type $\mathrm{II}^{45}$. The evolution of the shell has allowed tortoise to avoid having to increase locomotor speed to escape; however these adaptations for moving slowly and efficiently do not appear compatible with minimising the metabolic cost of self-righting. The rate at which muscles use metabolic energy increases sharply with increasing rate of contraction. Therefore, the rapid neck and limb movements during self-righting (Supplementary Movie $\mathrm{S} 1$ ), come at the cost of approximately doubling the $\mathrm{P}_{\text {met }}$ (relative to walking), which can be explained by the increase in oxygen consumption with increases in muscle contraction rate and the recruitment of faster twitch fibres ${ }^{45}$. The linear increase in $\mathrm{P}_{\text {met }}$ with the time taken to right (Fig. 1c) is an interesting phenomenon. It suggests that the power employed, in other words the effort that the animal makes, increases as the time the animal has spent inverted increases. This observation supports previous estimations using time as a proxy for energetic cost $t^{5}$.

\section{Conclusion}

Overcoming the dangers of inverting is a unique challenge faced by tortoises as armoured-bodied tetrapods. Our data indicate that self-righting requires a specific set of biomechanical movements to be successful and is a relatively metabolically costly behaviour in sub-adult female T. graeca. Sexual dimorphism in righting performance, with males righting faster and more often than females on average, has been documented in tortoises ${ }^{17}$; and it would be interesting to determine in future work if similar sex differences were found in the metabolic cost of self-righting. In sub-adult female T. graeca in our study the relatively high cost can be explained by the constraints of breathing upside down and the increased muscular work required for rapid limbs movements during self-righting. Research is needed on the frequency or risk of becoming inverted for any individual Testudines species. However, our data strengthen the links made between the fitness proxies associated with righting ability, and may ultimately allow us to better understand route choice and movement patterns. Our data would predict that in the wild, tortoises should eschew environments and behaviours where they are more prone to inverting to avoid the increased metabolic costs of turning right side up.

\section{Methods}

Animals. All experimental protocols were performed in accordance with the relevant guidelines and regulations and approved by the University of Manchester Research Governance, Ethics and Integrity Committee (Permit D.039). Animal care and experimentation were also in accordance with the ARRIVE guidelines. Captive born, 2-year-old female Mediterranean spur-thighed tortoises (Testudo graeca, $n=5$ ) were housed in the Biological Services Facility at the University of Manchester. Tortoises were maintained indoors in a vivarium $(2 \times 0.9 \times 0.7 \mathrm{~m})$ under UV and natural light bulbs, and ceramic heating lamps set up to provide a thermal gradient from hot $\left(35^{\circ} \mathrm{C}\right)$ to $\operatorname{cool}\left(25^{\circ} \mathrm{C}\right)$ regions ${ }^{46}$. Body mass was recorded at the start of trials (Electronic Supplementary Material, ESM). Fine-grain sand was selected as a substrate representative of natural conditions and tortoises were provided with rocks, wooden logs and tunnels for enrichment. Tortoises were fed a mix of commercial tortoise pellets (Komodo Complete Holistic Tortoise Diet, Happy Pets Products, Leicester, UK) with 
mixed fresh vegetables and dark leafy greens. Cuttlefish bones were also provided. Because tortoise digestion can take up to several weeks to months ${ }^{47}$, individuals were not fasted prior to experimentation. Food and water were available ad libitum.

Experimental protocol. Ectothermic physiological performance is temperature-dependent ${ }^{48}$, and optimal performance occurs around $35^{\circ} \mathrm{C}$ in reptiles ${ }^{49}$. Therefore, tortoises were maintained in the tank hot zone immediately prior to all experimentation and body temperature averaged $34.6 \pm 0.4{ }^{\circ} \mathrm{C}$ during all trials. The same tortoises were then examined under two separate experimental protocols to measure the CoR and the CoT. For the CoR trials a randomly selected tortoise was placed into the righting chamber $(40 \times 30 \times 20 \mathrm{~cm})$ filled with fine sand to a depth of $10 \mathrm{~cm}$ (Supplementary Movie S1). Once gas concentrations became stable during the resting period we measured a resting metabolic rate (RMR), then the chamber lid was then quickly lifted, the tortoise placed into an inverted position and the lid replaced. After self-righting the individual was then left in the chamber to settle and record a second RMR before removal. For the CoT trials, tortoises were trained for 4 months to walk inside a Perspex box $(20 \times 15 \times 10 \mathrm{~cm})$ on a small animal treadmill (Model: LE8710, Panlab, Harvard Apparatus, Spain). Preliminary trails indicated that the tortoises were restricted to a walking gait over a limited speed range (3-10 $\mathrm{cm} \mathrm{s}^{-1}$ ). When freely moving along a race during initial training the tortoises preferentially chose to move at mid-range speeds of 5-7 $\mathrm{cm} \mathrm{s}^{-1}$. Therefore, we selected a mid-range, easily sustainable speed ( $6 \mathrm{~cm} \mathrm{~s}^{-1}$, Supplementary Movie S2) for comparison to CoR. During walking trials, a randomly selected tortoise was placed onto the treadmill to record RMR, then walked at $6 \mathrm{~cm} \mathrm{~s}^{-1}$ until a steady trace was achieved (3-5 min) after which time a second RMR trace was recorded. Replicates for each tortoise were recorded for CoR and CoT of between 2 and 6 trials, and tortoises were given a rest day in between trials. For both CoR and CoT experiments, the lowest value was defined as RMR.

Respirometry and kinematics. Oxygen consumption $\left(\dot{V} \mathrm{O}_{2}\right)$ and carbon dioxide production $\left(\dot{V} \mathrm{CO}_{2}\right)$ were measured using an open-flow respirometry system (all equipment and software: Sable Systems International, Las Vegas, NV, USA). Air was pulled through the respirometry chamber at $2 \mathrm{~L} \mathrm{~min}^{-1}$ and $0.3 \mathrm{~L} \mathrm{~min}^{-1}$ (CoR and CoT, respectively) using a mass flow pump (MFS-2). Water vapour pressure of excurrent air was then measured (RH300 water vapour meter) before water was removed from the airstream using calcium chloride $(2-6 \mathrm{~mm}$ granular, Merck, Germany) after which $\mathrm{CO}_{2}$ content was measured (CA-10a $\mathrm{CO}_{2}$ analyser). Soda lime (2-5 mm granular, Sigma-Aldrich, Germany) was then used to scrub $\mathrm{CO}_{2}$ and finally $\mathrm{O}_{2}$ was measured (Oxzilla II absolute and differential dual channel $\mathrm{O}_{2}$ analyser). Each system was calibrated using a known flow rate of nitrogen, and found to be accurate to between $\pm 3-5 \%$ for both experimental set-ups. The primary flow rate was adjusted to a dry-corrected flow rate and oxygen consumption and carbon dioxide production calculated using the appropriate equations ${ }^{50}$. $\dot{V} \mathrm{CO}_{2}$ was then used with $V \mathrm{O}_{2}$ to calculate the respiratory exchange ratio $\left(\mathrm{RER}=\dot{V} C \mathrm{O}_{2}: \dot{V} \mathrm{O}_{2}\right)$. $\dot{V} \mathrm{O}_{2}$ was then converted to mass-specific power consumption $\left(\mathrm{P}_{\text {met }}, \mathrm{W} \mathrm{kg}^{-1}\right)$ by multiplying $\dot{V} \mathrm{O}_{2}$ by the calorific RER equivalent (in Joules) taken from (Table 12.1 ${ }^{51}$ ). Data were processed using ExpeData using a 60-s segment of a steady state trace during CoT and CoR trials. For analysis of total mass specific power over the CoR trial the entire steady state section of the trace was used. A Sony cyber-shot camera (DSC-RX-10 III, Sony Corporation", Tokyo, Japan) was used to film all trials at $100 \mathrm{fps}$ during self-righting and walking. Tracker v.2.51 (https://physl ets.org/tracker; The Open Source Physics Project) was used to analyse limb movement frequency (MF; Hz).

Data analysis. All statistical analyses (Supplementary Table S2) were performed using R statistical software (version 3.6.2, R Core Team 2020, http://www.R-project.org/). Differences in $\dot{V} \mathrm{O}_{2}$ and $\mathrm{P}_{\text {met }}$ between resting, walking, and righting tortoises, and frequency of limb movements between walking and righting tortoises, were examined using a one-way ANOVA with a post-hoc Tukey HSD test. The relationship between $\mathrm{P}_{\text {met }}$ and time (min), was investigated using a linear regression. Statistical differences were considered significant when $p<0.05$.

Received: 23 September 2021; Accepted: 20 December 2021

Published online: 10 January 2022

\section{References}

1. Lyson, T. R. et al. Origin of the unique ventilatory apparatus of turtles. Nat. Commun. 5(5211), 1-11. https://doi.org/10.1038/ ncomms6211 (2014)

2. Gans, C. \& Hughes, G. The mechanism of lung ventilation in the tortoise Testudo graeca Linné. J. Exp. Biol. 47(1), 1-20 (1967).

3. Jackson, D. C., Singer, J. H. \& Downey, P. T. Oxidative cost of breathing in the turtle Chrysemys picta bellii. Am. J. Physiol. 261, R1325-R1328 (1991).

4. Landberg, T., Mailhot, J. D. \& Brainerd, E. L. Lung ventilation during treadmill locomotion in a semi-aquatic turtle, Trachemys scripta. J. Exp. Zool. 311A, 551-562. https://doi.org/10.1002/jez.478 (2009).

5. Ruhr, I., Rose, K., Sellers, W., Crossley, D. II. \& Codd, J. Turning turtle: Scaling relationships and self-righting ability in Chelydra serpentina. Proc. R. Soc. B. 288, 20210213. https://doi.org/10.1098/rspb.2021.0213 (2021).

6. Pritchard, P. C. H. Encyclopaedia of Turtles (TFH, 1979).

7. Carr, A. Handbook of Turtles: The Turtles of the United States, Canada, and Baja California (Cornell University Press, 1952).

8. Rivera, G. Ecomorphological variation in shell shape of the freshwater turtle Pseudemys concinna inhabiting different aquatic flow regimes. Int. Comp. Biol. 48(6), 769-787. https://doi.org/10.1093/icb/icn088 (2008).

9. McNeill Alexander, R. Gaits of mammals and turtles. J. R. Soc. Jpn. 11(3), 314-319 (1993).

10. Zani, P. A. \& Kram, R. Low metabolic cost of locomotion in ornate box turtles, Terrapene ornate. J. Exp. Biol. 211, 3671-3676. https://doi.org/10.1242/jeb.019869 (2008). 
11. Sellers, W. I., Rose, K. A. R., Crossley, D. A. II. \& Codd, J. R. Inferring cost of transport from whole-body kinematics in three sympatric turtle species with different locomotor habits. Comp. Biochem. Physiol. A. 247, 110739. https://doi.org/10.1016/j.cbpa. 2020.110739 (2020).

12. Chiari, Y., van der Meijden, A., Caccone, A., Claude, J. \& Gilles, B. Self-righting potential and the evolution of shell shape in Galápagos tortoises. Sci. Rep. 7(1), 1-8. https://doi.org/10.1038/s41598-017-15787-7 (2017).

13. Woledge, R. C. The energetics of tortoise muscle. J. Physiol. 197(3), 685-707 (1968).

14. Steyermark, A. C. \& Spotila, J. R. Body temperature and maternal identity affect snapping turtle (Chelydra serpentina) righting response. Copeia 4, 1050-1057. https://doi.org/10.1643/0045-8511(2001)001[1050:BTAMIA]2.0.CO;2 (2001).

15. Rubin, A. M., Blob, R. W. \& Mayerl, C. J. Biomechanical factors influencing successful self-righting in the Pleurodire turtle, Emydura subglobosa. J. Exp. Biol. 221, jeb182642. https://doi.org/10.1242/jeb.182642 (2018).

16. Penn, D. \& Brockmann, H. J. Age-biased stranding and righting in male horseshoe crabs, Limulus polyphemus. Anim. Behav. 49, 1531-1539. https://doi.org/10.1016/003-3472(95)90074-8 (1995).

17. Bonnet, X. et al. Sexual dimorphism in steppe tortoises (Testudo horsfieldii): Influence of the environment and sexual selection on body shape and mobility. Biol. J. Linn. Soc. 72, 357-372. https://doi.org/10.1006/bjls.2000.0504 (2001).

18. Zuffi, M. A. L. \& Corti, C. Aspects of population ecology of Testudo hermanni hermanni from Asinara Island, NW Sardinia (Italy, Western Mediterranean Sea): Preliminary data. Amphib-Reptil. 24, 441-447 (2003).

19. Domokos, G. \& Várkonyi, P. L. Geometry and self-righting of turtles. Proc. R. Soc. B. 275(1630), 11-17. https://doi.org/10.1098/ rspb.2007.1188 (2008)

20. Mann, G. K. H., O’Riain, M. J. \& Hofmeyr, M. D. Shaping up to fight: Sexual selection influences body shape and size in the fighting tortoise (Chersina angulata). J. Zool. 269, 373-379. https://doi.org/10.1111/j.1469-7998.2006.00079x (2006).

21. Golubović, A., Bonnet, X., Djordjević, S., Djurakic, M. \& Tomović, L. Variations in righting behavior across Hermann’s tortoise populations. J. Zool. 291, 69-75. https://doi.org/10.1111/jzo.12047 (2013).

22. Golubović, A., Andelkovic, M., Arsovski, D., Bonnet, X. \& Tomović, L. Locomotor performances reflect habitat constraints in an armoured species. Behav. Ecol. Sociobiol. 71, 93. https://doi.org/10.1007/s00265-017-2318-0 (2017).

23. Ashe, V. M. The righting reflex in turtles: A description and comparison. Psychol. Sci. 20, 150-152. https://doi.org/10.3758/BF033 35647 (1970).

24. Golubović, A., Tomović, L. \& Ivanović, A. Geometry of self-righting: The case of Hermann’s tortoises. Zool. Anz. 254, 99-105. https://doi.org/10.1016/j.jcz.2014.12.003 (2015).

25. Finkler, M. S. Influence of water availability during hatching on hatchling size, body composition, desiccation tolerance, and terrestrial locomotor performance in the snapping turtle, Chelydra serpentina. Physiol. Biochem. Zool. 72, 714-722. https://doi.org/ 10.1086/316711 (1999).

26. Stojadinović, D., Milošević, D. \& Crnobrnja-Isailović, J. Righting time versus shell size and shape dimorphism in adult Hermann’s tortoises: Field observations meet theoretical predictions. Anim. Biol. 63(4), 381-396. https://doi.org/10.1163/15707563-00002 420 (2013).

27. Delmas, V., Baudry, E., Girondot, M. \& Prevot-Julliard, A.-C. The righting reflex as a fitness indicator in freshwater turtles. Biol. J. Linn. Soc. 91, 99-109. https://doi.org/10.1111/j.1095-8312/2007.00780.x (2007).

28. Burger, J. Behavior of hatchling diamondback terrapins (Malaclemys terrapin) in the field. Copeia 1976, 742. https://doi.org/10. $2307 / 1443457$ (1976).

29. Landberg, T., Mailhot, J. D. \& Brainerd, E. L. Lung ventilation during treadmill locomotion in a terrestrial turtle, Terrapene carolina. J. Exp. Biol. 206, 3391-3404. https://doi.org/10.1242/jeb.00553 (2003).

30. Gaunt, A. S. \& Gans, C. Mechanics of respiration in the snapping turtle, Chelydra serpentina (Linné). J. Morph. 128, 195-227. https://doi.org/10.1002/jmor.1051280205 (1969).

31. Lambertz, M., Böhme, W. \& Perry, S. F. The anatomy of the respiratory system in Platysternon megacephalum Gray, 1831 (Testudines: Crytodira) and related species, and its phylogenetic implications. Comp. Biochem. Physiol. 156, 330-336. https://doi.org/ 10.1016/j.cbpa.2009.12.016 (2010).

32. de Souza, R. B. B. \& Klein, W. The influence of the post-pulmonary septum and submersion on the pulmonary mechanics of Trachemys scripta (Cryptodira: Emydidae). J. Exp. Biol. 224(12), 242386. https://doi.org/10.1242/jeb.242386 (2021).

33. Jodice, P. G. R., Epperson, D. M. \& Visser, G. H. Daily energy expenditure in free-ranging gopher tortoises (Gopherus polyphemus). Copeia 2006(1), 129-136. https://doi.org/10.1643/0045-8511(2006)006[0129:DEEIFG]2.0.CO;2 (2006).

34. Zera, A. J. \& Harshman, L. G. The physiology of life history trade-offs in animals. Ann. Rev. Ecol. Syst. 32, 95-126. https://doi.org/ 10.1146/annurev.ecolsys.32.081501.114006 (2001).

35. Shadmehr, R., Huang, H. J. \& Ahmed, A. A. A representation of effort in decision-making and motor control. Curr. Biol. 26, 1929-1934. https://doi.org/10.1016/j.cub.2016.05.065 (2016).

36. Shepard, E. L. C. et al. Energy landscapes shapes animal movement ecology. Am. Nat. 182(3), 298-312. https://doi.org/10.1086/ 671257 (2013)

37. Baudinette, R. V., Miller, A. M. \& Sarre, M. P. Aquatic and terrestrial locomotory energetics in a toad and a turtle: A search for generalisations among ectotherms. Physiol. Biochem. Zool. 73(6), 672-682. https://doi.org/10.1086/318101 (2000).

38. Hailey, A. \& Coulson, I. M. Measurement of time budgets from continuous observation of thread-trailed tortoises (Kinixys spekii). Herp. J. 9, 15-20 (1999).

39. Kram, R. \& Taylor, C. R. Energetics of running: A new perspective. Nature 346, 265-267. https://doi.org/10.1038/346265a0 (1990).

40. Taylor, C. R. Relating mechanics and energetics during exercise. Adv. Vet. Sci. Comp. Med. 38A, 181-215 (1994).

41. Cavagna, G. A. \& Kaneko, M. Mechanical work and efficiency in level walking and running. J. Physiol. 268(2), 467-481. https:// doi.org/10.1113/jphysiol.1977.sp011866 (1977).

42. Carrier, D. R., Deban, S. M. \& Fischbein, T. Locomotor function of the pectoral girdle "muscular sling" in trotting dogs. J. Exp. Biol. 209, 2224-2237. https://doi.org/10.1242/jeb.02236 (2006).

43. Heglund, N. C. \& Cavagna, G. A. Efficiency of vertebrate locomotory muscles. J. Exp. Biol. 115, 283-292. https://doi.org/10.1242/ jeb.115.1.283 (1985).

44. Barclay, C. J. The basis of difference in thermodynamic efficiency among skeletal muscles. Clin. Exp. Pharm. Physiol. 44(12), 1279-1286. https://doi.org/10.1111/1440-1681.12850 (2017).

45. Nwoye, L. O. \& Goldspink, G. Biochemical efficiency and intrinsic shortening speed in selected fast and slow muscles. Experientia 37, 856-857. https://doi.org/10.1007/BF1985678 (1981).

46. Lambert, M. Temperature, activity and field sighting in the Mediterranean spur-thighed or common garden tortoise Testudo graeca. Biol. Conserv. 21, 39-54. https://doi.org/10.1016/0006-3207(81)90067-7 (1981).

47. Tracy, R., Zimmerman, L., Tracy, C., Bradley, K. \& Castle, K. Rates of food passage in the digestive tract of young desert tortoises: Effects of body size and diet quality. Chelonian Conserv. Biol. 5(2), 269-273. https://doi.org/10.2744/1071-8443(2006)5[269:ROFPIT]2.0.co;2 (2006).

48. Huey, R. \& Kingsolver, J. Evolution of thermal sensitivity of ectotherm performance. Trends Ecol. Evol. 4(5), 131-135. https://doi. org/10.1016/0169-5347(89)90211-5 (1989).

49. Lailvaux, S. \& Irschick, D. Effects of temperature and sex on jump performance and biomechanics in the lizard Anolis carolinensis. Funct. Ecol. 21(3), 534-543. https://doi.org/10.1111/j.1365-2435.2007.01263.x (2007)

50. Lighton, J. Measuring Metabolic Rates: A Manual for Scientists (Oxford University Press, 2008). 
51. Brody, S. Bioenergetics and Growth (Reinhold, 1945).

\section{Acknowledgements}

We would like to thank Andrés Mármol-Guijarro and Ilan Ruhr who provided valuable assistance in animal husbandry and training. This work was supported by The Leverhulme Trust (RPG-2019-104), the BBSRC (BB/ I021116/1) and the NSF (17565187).

\section{Author contributions}

J.C., D.A.C., and W.I.S. conceived and designed the study. J.C., W.S., and D.C. secured the funding. H.E., M.L., and P.T. collected the data and performed the formal analysis: H.E., P.T., M.L., W.S., D.C., and J.C. wrote the manuscript. All authors reviewed and edited the manuscript.

\section{Competing interests}

The authors declare no competing interests.

\section{Additional information}

Supplementary Information The online version contains supplementary material available at https://doi.org/ 10.1038/s41598-021-04273-w.

Correspondence and requests for materials should be addressed to J.R.C.

Reprints and permissions information is available at www.nature.com/reprints.

Publisher's note Springer Nature remains neutral with regard to jurisdictional claims in published maps and institutional affiliations.

Open Access This article is licensed under a Creative Commons Attribution 4.0 International License, which permits use, sharing, adaptation, distribution and reproduction in any medium or format, as long as you give appropriate credit to the original author(s) and the source, provide a link to the Creative Commons licence, and indicate if changes were made. The images or other third party material in this article are included in the article's Creative Commons licence, unless indicated otherwise in a credit line to the material. If material is not included in the article's Creative Commons licence and your intended use is not permitted by statutory regulation or exceeds the permitted use, you will need to obtain permission directly from the copyright holder. To view a copy of this licence, visit http://creativecommons.org/licenses/by/4.0/.

(C) The Author(s) 2022 